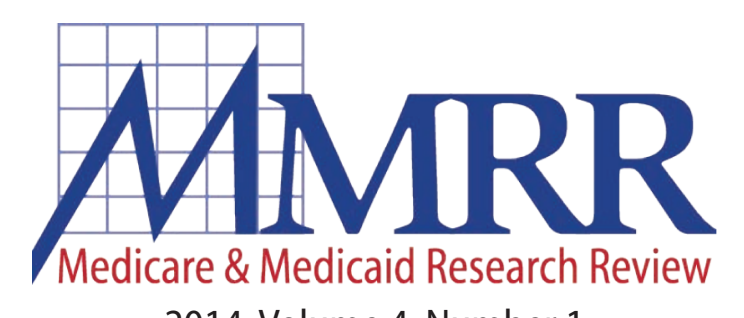

2014: Volume 4, Number 1

A publication of the Centers for Medicare \& Medicaid Services, Office of Information Products \& Data Analytics

\title{
Medicare-Medicaid Eligible Beneficiaries and Potentially Avoidable Hospitalizations
}

\author{
Misha Segal, ${ }^{1}$ Eric Rollins, ${ }^{1}$ Kevin Hodges, ${ }^{1}$ and Michelle Roozeboom ${ }^{2}$ \\ ${ }^{1}$ Centers for Medicare \& Medicaid Services \\ ${ }^{2}$ General Dynamics Information Technology
}

Objective: Potentially avoidable hospitalizations have been identified by experts as leading to poor health outcomes and costly care. Potentially avoidable hospitalizations are particularly common among full-benefit dual eligible beneficiaries. This paper examines potentially avoidable hospitalizations rates by setting, state, and medical condition, and the average cost of these events.

Methods: This analysis identifies potentially avoidable hospitalizations using diagnosis codes identified by an expert panel. Settings of care are determined using a timeline file, which assigns an individual to a specific setting on a particular day.

Population/Data Source: The analysis uses several different datasets from the Chronic Conditions Data Warehouse. The study population includes fee-for-service beneficiaries who were eligible for both Medicare and full Medicaid benefits for at least one month during the calendar year. The study years are 2007 to 2009.

Results: In 2009, among our study population, 26 percent of hospitalizations were potentially avoidable; and the rate was 133 per 1,000 person-years. Potentially avoidable hospitalizations were much more likely for those beneficiaries who were in institutions-16 percent of beneficiaries in our study population were in an institution, yet comprised 45 percent of all potentially avoidable hospitalizations. The range in rates across the states was considerable, with more than a threefold difference across states. Five conditions were responsible for nearly 80 percent of potentially avoidablehospitalizations. From 2007 to 2009, the national and state rates were fairly consistent.

Discussion: This analysis indicates that the potentially avoidable hospitalization rate among MME beneficiaries was consistently high from 2007 to 2009. This bears monitoring in the future to see if the Centers for Medicare \& Medicaid Services' various initiatives have led to a reduction in rates.

Keywords: Dual Eligibles/MMEs, Potentially avoidable hospitalizations, quality metrics, readmissions, Medicaid, Medicare, quality of care, patient safety (measurement)

ISSN: 2159-0354

doi: http://dx.doi.org/10.5600/mmrr.004.01.b01 


\section{Medicare \& Medicaid Research Review} 2014: Volume 4, Number 1

\section{Mission Statement}

Medicare \& Medicaid Research Review is a peerreviewed, online journal reporting data and research that informs current and future directions of the Medicare, Medicaid, and Children's Health Insurance programs. The journal seeks to examine and evaluate health care coverage, quality and access to care for beneficiaries, and payment for health services.

\section{http://www.cms.gov/MMRR/}

\section{U.S. Department of Health \& Human Services Kathleen Sebelius Secretary}

\section{Centers for Medicare \& Medicaid Services Marilyn Tavenner Administrator}

Editor-in-Chief

David M. Bott, Ph.D.

The complete list of Editorial Staff and Editorial Board members

may be found on the MMRR Web site (click link): $\underline{\text { MMRR Editorial Staff Page }}$

Contact: $\underline{\text { mmrr-editors@cms.hhs.gov }}$

Published by the Centers for Medicare \& Medicaid Services.

All material in the Medicare \& Medicaid Research

Review is in the public domain and may be duplicated without permission. Citation to source is requested.

\section{Introduction}

Potentially avoidable hospitalizations are hospitalizations that could have been avoided because the condition could have been prevented or treated outside of an inpatient hospital setting. Reducing the number of these events has been identified as a promising way to improve care and lower health care costs. Potentially avoidable hospitalizations are particularly common among dual eligible beneficiaries, referred to in this brief as Medicare and Medicaid enrollees (MMEs).

MMEs qualify for both Medicare and Medicaid benefits and represent approximately 20 percent of the total Medicare population (Centers for Medicare \& Medicaid Services, 2013a). In 2009, 7.1 million of the 9.3 million MMEs received full benefits from both Medicare and Medicaid for one month or more (Centers for Medicare \& Medicaid Services, 2013a).

MMEs are typically seniors or those under the age of 65 with disabilities and are generally poorer and have worse health status than other Medicare beneficiaries. On average, they have 25 percent more chronic conditions than non-MMEs, based on 15 conditions. ${ }^{1}$ In particular, MMEs were much more likely to have depression, Alzheimer's disease, diabetes, heart failure, chronic kidney disease, chronic obstructive pulmonary disease (COPD), asthma, or stroke. They account for a disproportionate share of Medicare spending (Coughlin, Waidmann, \& Phadera, 2012).

MMEs were more likely to have both a hospitalization and a potentially avoidable hospitalization than the average Medicare

\footnotetext{
${ }^{1}$ The authors performed this analysis for the following 15 conditions: Alzheimer's disease, arthritis, asthma, atrial fibrillation, cancer, chronic kidney disease, COPD, depression, diabetes, heart failure, hyperlipidemia, hypertension, ischemic heart disease, osteoporosis, and stroke.
} 
beneficiary. In 2009, 26 percent of MMEs had at least one hospitalization, compared to 20 percent of the entire Medicare fee-for-service population, and nine percent of MMEs had at least one potentially avoidable hospitalization, compared to four percent of the overall Medicare population (Centers for Medicare \& Medicaid Services, 2013b).

Previous research found that 26 percent of hospitalizations for MMEs in 2005 were potentially avoidable, at a cost of $\$ 5.6$ billion (Segal, 2011). This study updates those findings with 2007 to 2009 data, and examines differences in potentially avoidable hospitalization rates by setting, state, and medical condition. ${ }^{2}$

\section{Population/Data Source}

The analysis uses several different datasets from the Chronic Conditions Data Warehouse (CCW): complete Medicare fee-for-service (FFS) claims and eligibility data, complete Medicaid FFS claims and eligibility data from the Medicaid Analytic Extract (MAX), the CCW's predefined chronic condition flags, and the Medicare timeline file (which is created using Medicare claims and assessment data from the Minimum Data Set and the Outcome and Assessment Information Set).

The study population is beneficiaries who were eligible for both Medicare and full Medicaid benefits for at least one month during the calendar year. We excluded MMEs from this analysis if they were enrolled in a managed care plan (either Medicare, Medicaid, or both) at any point during a calendar year. In 2009, after these exclusions, 73 percent of all MMEs were included in the study population (5.1 million of 7.1 million). The proportion of MMEs included in the study population was slightly higher in 2007 (75 percent) and 2008 (74 percent).

${ }^{2}$ The results from this study are not directly comparable to the previous research paper due to methodological differences.

\section{Methods}

\section{Potentially Avoidable Hospitalization Algorithm}

This study identified potentially avoidable hospitalizations using the same logic and diagnosis codes described in Walsh, Freiman, Haber, Bragg, Ouslander, \& Wiener (2010). The algorithm was developed by a technical expert panel that was sponsored by the Centers for Medicare \& Medicaid Services (CMS) and included experts in geriatrics, ambulatory care sensitive conditions, and other quality measures. The algorithm also drew on previous work on potentially avoidable hospitalizations for those in ambulatory settings and nursing facilities (Carter, 2003; Grabowski, O’Malley, \& Barhydt, 2007; Maslow \& Ouslander, 2012; Torio, Elixhauser, \& Andrews, 2013).

The primary diagnosis code on an inpatient hospital claim was used to identify potentially avoidable hospitalizations, although the list of diagnoses that were considered differed by setting. The technical expert panel concluded that certain conditions could be handled appropriately in an institution, such as a nursing home, but may lead to an unavoidable hospitalization for beneficiaries living in the community (Walsh, Freiman, Haber, Bragg, Ouslander, \& Wiener, 2010). These conditions include pneumonia, skin ulcers, diarrhea, and falls.

The algorithm includes a list of 16 conditions that could result in potentially avoidable hospitalizations for individuals in institutional settings; nine of these conditions also apply to individuals who are living in the community, receiving Medicare home health, or receiving Medicaid home- and community-based waiver services. The nine conditions that apply to all settings are: $\mathrm{COPD} /$ asthma, congestive heart failure (CHF), constipation, dehydration, hypertension, poor glycemic control, seizures, urinary tract 
infection, and weight loss/malnutrition. The seven additional conditions that apply only to institutional settings are: altered mental status, anemia, diarrhea, falls/trauma, pneumonia, psychosis/agitation, and skin ulcers.

We used the following ICD-9 diagnosis codes to identify a potentially avoidable hospitalization:

\section{Conditions for both institutional and non-institutional settings}

- COPD, chronic bronchitis, and asthma: 466-466.99, 490-490.99, 491.0, 491.1, 491.2, 491.20, 491.21, 491.8, 491.9, 492.0, 492.8, 493.00, 493.01, 493.02, 493.10, 493.11, 493.12, 493.20, 493.21, 493.22, 493.81, 493.82, 493.90, 493.91, 493.92, 494-494.99, 496-496.99

- Congestive heart failure: 398.91, 402.11, 402.91, 404.11, 404.13, 404.91, 404.93, 428.0, $428.1,428.20,428.21,428.22,428.23,428.30$, $428.31,428.32,428.33,428.40,428.41,428.42$, $428.43,428.9,518.4$

- Constipation, fecal impaction, and obstipation: 564.0, 564.00, 564.01, 564.09, 560.39

- Dehydration, volume depletion including acute renal failure and hyponatremia: 276.1, 276.5, 276.8, 584.0-584.99, 588.8, 588.81, $588.89,588.9$

- Hypertension and hypotension: 401.9, 402.10, 402.90, 403.10, 403.90, 404.10, 404.90, 458.0, 458.1, 458.2, 458.21, 458.29, 458.8, 458.9

- Poor glycemic control: 251.2, 250.2-250.29, 250.3-250.39, 250.1-250.19, 251.0, 250.02, $250.03,790.29$

- Seizures: 345-345.99, 436-436.99, 780.3780.39

- Urinary tract infection: 590.10, 590.11, 590.81, 590.9, 595.0, 595.1, 595.2, 595.4, 590.80, 595.89, 595.9, 597.0, 598.0-598.09, 599.0, 601-601.99

- Weight loss (failure to thrive) and nutritional deficiencies: 260-260.99, 261-261.99, 262262.99, 263-263.99, 268.0, 268.1, 783.21, $783.22,783.3,783.7$

\section{Conditions for institutional settings only}

- Altered mental status, acute confusion, and delirium: 290.3, 290.41, 292.81, 293, 293.0, 293.1

- Anemia: 280.0-280.99, 281.0-281.99, 285.20$285.29,285.9$

- Diarrhea, gastroenteritis, and C.difficile: 003.0, 004-004.99, 005-005.99, 006.0, 007.0007.99, 008-008.99, 009-009.99, 558.9, 787.91

- Falls and trauma: 800-805.08, 805.10, 805.11, $805.12,805.13,805.14,805.15,805.16,805.17$, $805.18,805.3,805.5,805.6,805.7,805.9$, $806-829.99,830-839.99,850-852.00,852.03$, $852.04,852.10,852.12,852.13,852.14,852.15$, $852.16,852.19,852.20-853.00,853.03,853.04$, 853.09-854.99, 905-905.99, 906-906.99, 907907.99, 908-908.99, 909-909.99, 925-929.99, 940-949.99, 958.9-959.99, 991.6-991.99, 992.0-992.99, 993.5-993.79, 994.1-994.39, 994.7-994.89

- Pneumonia and bronchitis (lower respiratory illness): 480-480.99, 481-481.99, 482-482.99, 483.0-483.9, 485-485.99, 486-486.99, 507.0

- Psychosis, severe agitation, and organic brain syndrome: 290.42, 290.43, 290.8, 290.9, 293.81, 293.82, 293.83, 293.84, 293.89, 293.9, 295.2, 297-297.99, 298-298.99

- Skin ulcers and cellulitis: 681-681.99, 682-682.99, 683-683.99, 686-686.99, 707.0$707.09,707.1-707.19,707.8,707.9$

\section{Settings of care and day counts}

For each potentially avoidable hospitalization, we examined the beneficiary's information in the Medicare timeline file to identify the setting when the admission occurred, and used that information to determine the prevalence of potentially avoidable hospitalizations by setting. We supplemented the Medicare timeline file with Medicaid waiver coverage information using the MAX personal summary file, and determined which inpatient care occurred in an inpatient psychiatric facility (IPF), 
inpatient rehabilitation facility (IRF), or long-term care hospital (LTCH) using the Medicare Part A claims file.

Our modified timeline file is hierarchical for each day. The order is: inpatient hospital stay, skilled nursing facility, LTCH, IRF, IPF, nursing home, Medicaid home- and community-based waiver, home health, and other/community. Since the MAX personal summary file only includes monthly waiver data, we assume that a beneficiary has waiver coverage for the entire month, other than days that are above it in the hierarchy (or due to death). We only included the days in which beneficiaries were eligible for both Medicare and full Medicaid benefits.

\section{Potentially avoidable hospitalization rate}

The rate at which potentially avoidable hospitalizations occur is expressed as the number per 1,000 person-years and, like a prevalence rate, is used because the length of stay varies across settings. As a simplified example, the potentially avoidable hospitalization rate for those using home health is
300 per 1,000 person-years. During the course of a year, if 10 MMEs received home health for the entire year, they would have a combined average of three potentially avoidable hospitalizations.

\section{Hospital costs}

Hospital costs include all Medicare Part A, Medicare Part B, and Medicaid costs during the hospital stay.

\section{Results}

In 2009, the national rate among MMEs for potentially avoidable hospitalizations was 133 per 1,000 person-years, but there was considerable variation across health care settings (see Exhibit 1). The rate was highest for those living in institutions. At any given point in time, one in six beneficiaries (16 percent) in our study population was in an institution, yet these enrollees comprised slightly more than 25 percent of all hospitalizations and about 45 percent of all potentially avoidable hospitalizations.

\section{Exhibit 1. 2009 Potentially Avoidable Hospitalizations Rates, by Setting of Care}

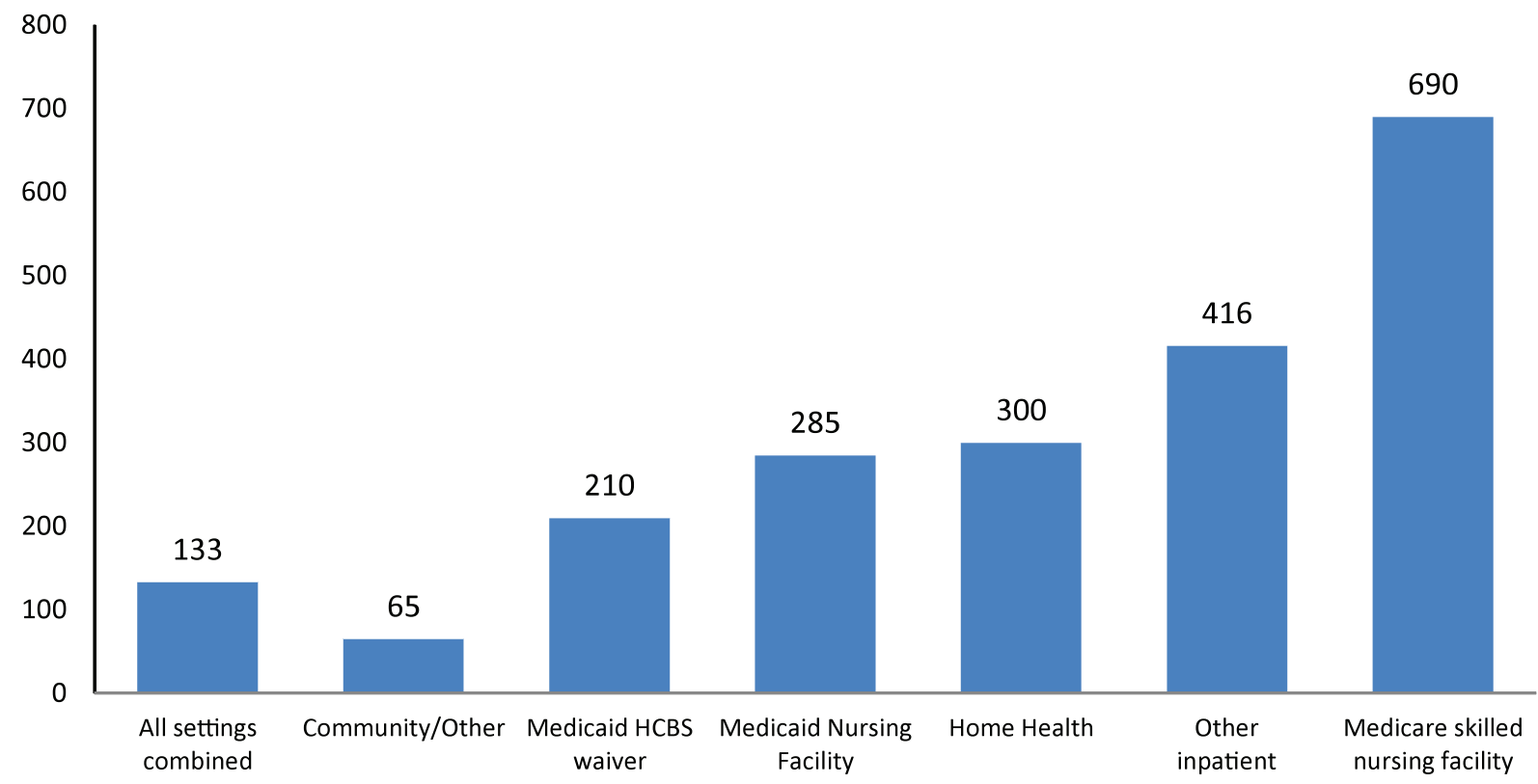

SOURCE: Chronic Condition Warehouse, 2009. 
More than 90 percent of institutionalized MME beneficiaries were in nursing homes. For those MMEs receiving care in a skilled nursing facility, the potentially avoidable hospitalizations rate was 690 per 1,000 person-years. For all other MMEs residing in nursing homes, the rate was 285 per 1,000 personyears. The combined rate for all other institutional settings of care-IRFs, LTCHs, IPFs, and cancer hospitals-was 416 per 1,000 person-years.

For MMEs in non-institutional settings, the potentially avoidable hospitalizations rate was lower. Beneficiaries who were enrolled in a waiver program for Medicaid home- and communitybased services (HCBS) had a rate of 210 per 1,000 person-years. For those who were receiving Medicare home health benefits, but were not enrolled in an HCBS waiver program, the rate was 300 per 1,000 person years.

The remaining beneficiaries, who were (1) not in an institution and (2) not receiving Medicaid HCBSor Medicarehomehealth benefits, constituted nearly 75 percent of the study population at any given point in time. The potentially avoidable hospitalization rate for these "Community/Other" MME beneficiaries was 65 per 1,000 person-years.

While differences in rates across settings are important for identifying strategies to reduce potentially avoidable hospitalizations, a few issues are worth highlighting when interpreting these differences. Beneficiaries typically spend far fewer days in skilled nursing facilities (SNFs) and "other inpatient" settings, such as IRFs, LTCHs, and IPFs, than in other settings due to the key role those inpatient settings play in stabilizing and rehabilitating complex patients. These rates do not adjust for the generally higher acuity levels for beneficiaries in these settings. As a result, it is not surprising that these settings have higher rates.

Among the almost 2.3 million hospitalizations for MMEs in 2009, over 600,000 (or 26 percent) were considered potentially avoidable, with an average cost of $\$ 8,783$ (Exhibit 2). The overall

\section{Exhibit 2. Percent of Potentially Avoidable Hospitalizations (PAH) and Associated Spending}

There were over 600,000 PAHs among the MME community in 2009. Total spending for these hospitalizations was over \$5.4 billion.

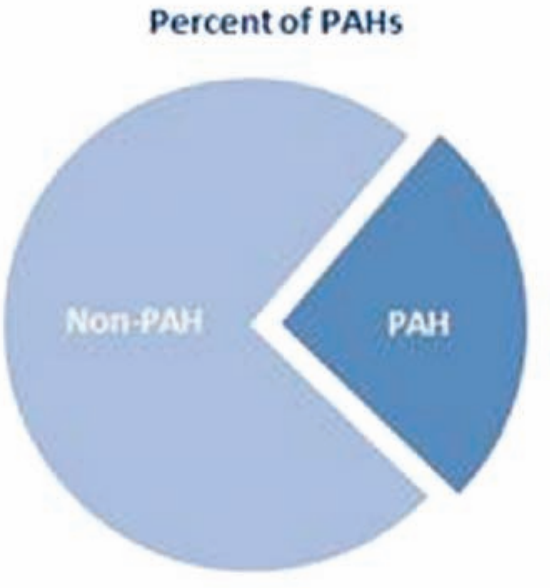

$26 \%$ of hospitalizations for MMEs in 2009 were PAHs.
PAH Spending

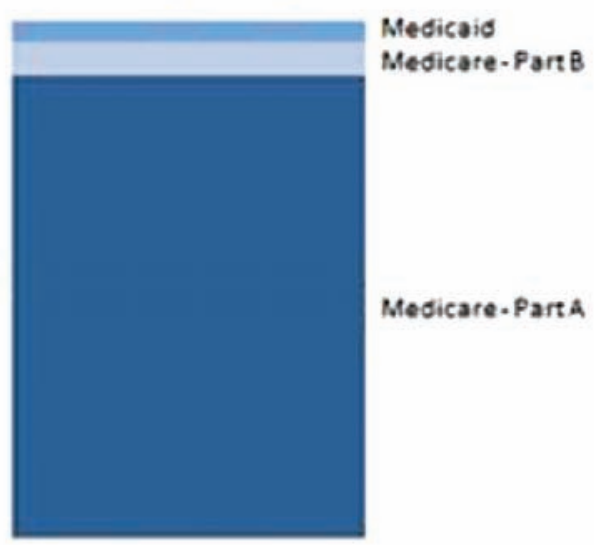

$96 \%$ of costs related to these

PAHs were paid for by Medicare.

SOURCE: Chronic Condition Warehouse, 2009. 
Medicare and Medicaid costs for these potentially avoidable hospitalizations were $\$ 5.4$ billion, with the Medicare program bearing 96 percent of the costs. Almost the entire Medicare portion (93 percent) was paid for through the Part A benefit, which covers inpatient hospitalization services. Medicare Part B, which covers physician services, paid for the remaining seven percent.

The range in potentially avoidable hospitalization rates across states is wide (see Exhibit 3). There is more than a threefold difference across states, from the lowest in Utah (59 per 1,000 person-years) to the highest in Mississippi (197 per 1,000 person years). The data show significant variation across states, but this study does not control for differences across states in patients' health status or other demographic characteristics, which may contribute to some of the observed differences.

Five conditions were responsible for nearly 80 percent of potentially avoidable hospitalizations occurring among the study population: congestive heart failure (21 percent), COPD/asthma (20 percent), urinary tract infections (15 percent), pneumonia (13 percent), and dehydration (11 percent; see Exhibit 4). There were differences in conditions by setting, in part due to definitional differences, because institutions have more conditions that are associated with potentially avoidable hospitalizations.

Pneumonia was the leading cause of potentially avoidable hospitalizations for those MMEs residing in institutional settings, accounting for nearly 30 percent of all potentially avoidable hospitalizations. Urinary tract infections and dehydration were also leading causes. However, there were some differences across institutional settings: falls/ trauma accounted for a significantly higher proportion of potentially avoidable hospitalizations for those residing in nursing homes than for those receiving care in other institutions.

For beneficiaries who were in a Medicaid HCBS program, receiving Medicare home health

Exhibit 3. Potentially Avoidable Hospitalization (PAH) Rates by State for 2009.

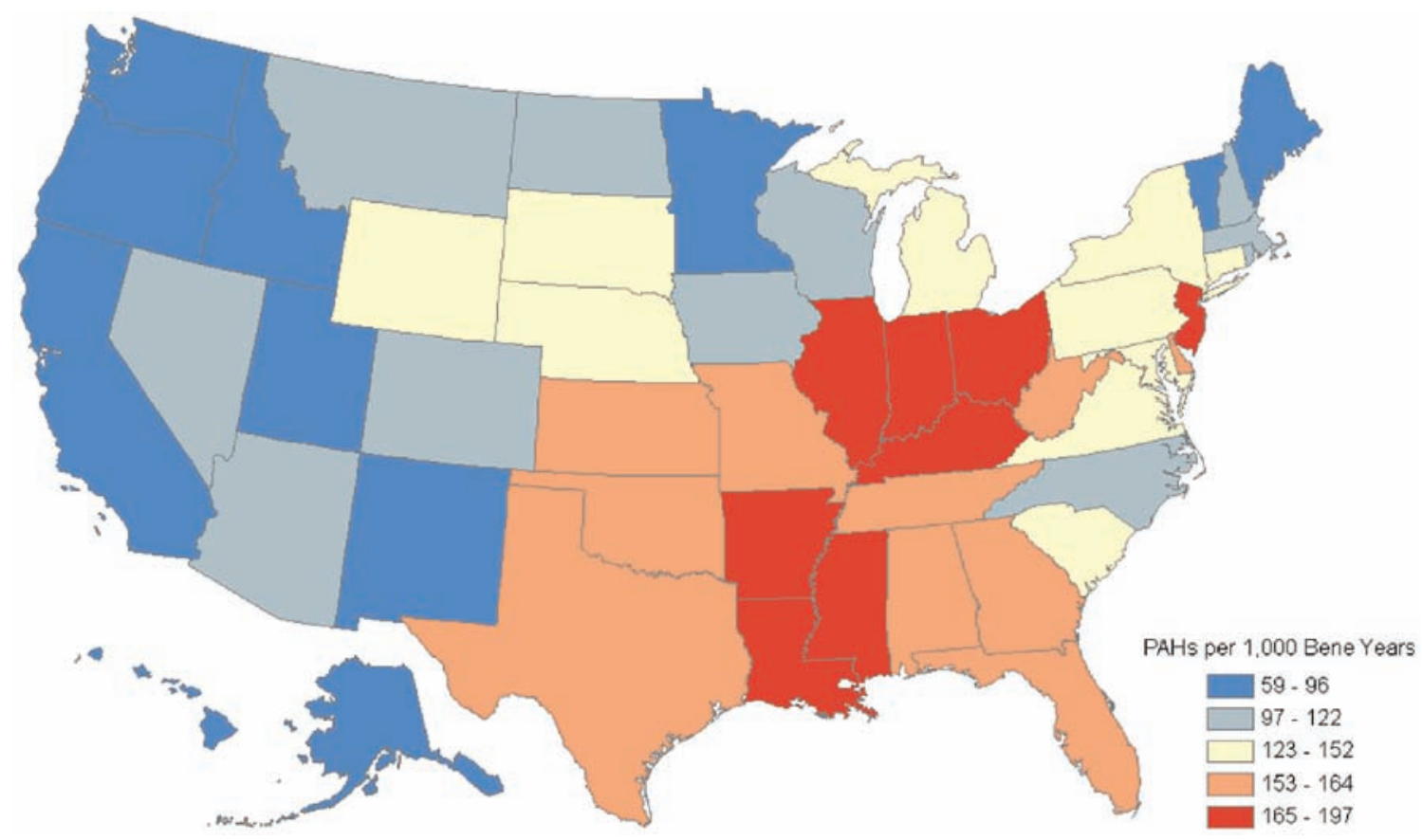

SOURCE: Chronic Condition Warehouse, 2009. 
Exhibit 4. Highly Prevalent Conditions Associated with Potentially Avoidable Hospitalizations in 2009

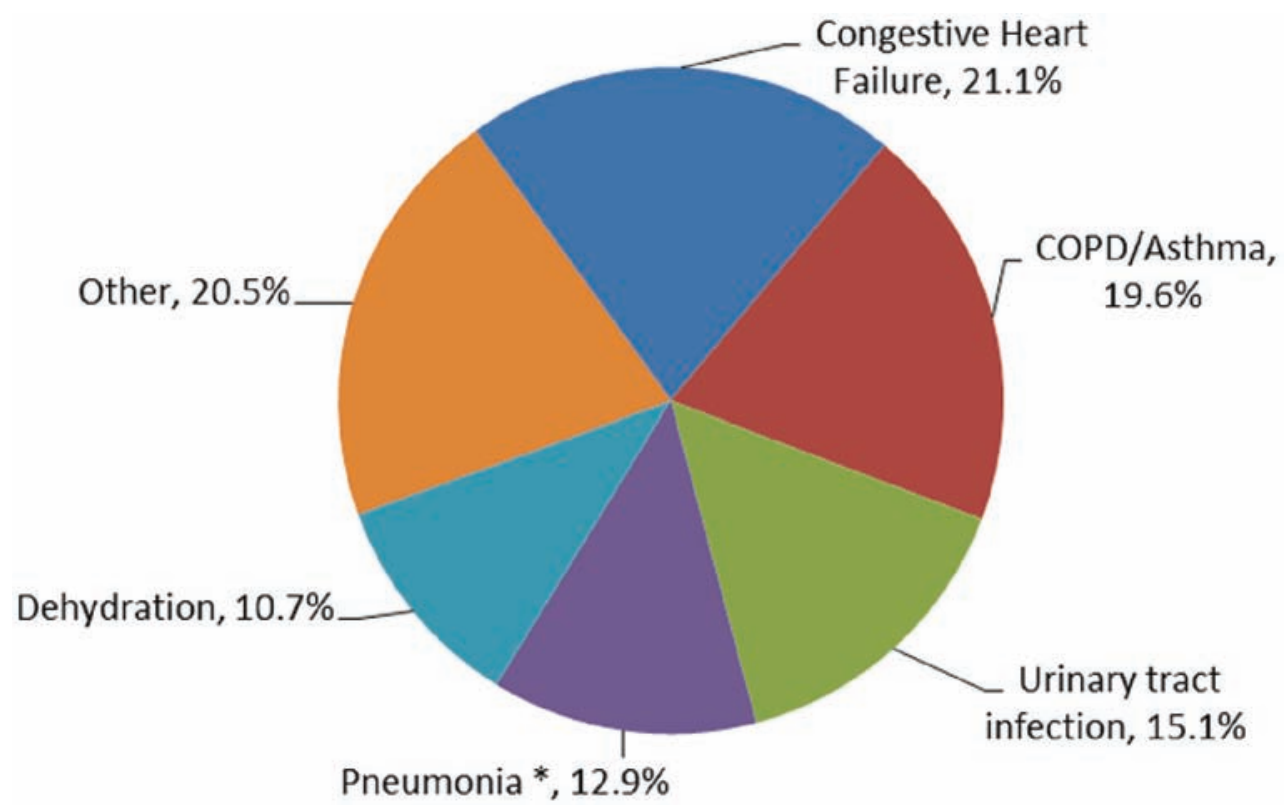

NOTE: * Pneumonia was only considered as the primary diagnosis for a potentially avoidable hospitalizations for beneficiaries in institutions. SOURCE: Chronic Condition Warehouse, 2009.

benefits, or otherwise living in the community, congestive heart failure and $\mathrm{COPD} /$ asthma accounted for approximately 60 percent of all potentially avoidable hospitalizations.

From 2007 to 2009 , the state rates for potentially avoidable hospitalizations stayed relatively consistent. For MMEs, the national rates were flat between 2007 and 2009, with rates of 134, 136, and 133 per 1,000 person-years, respectively. State rankings also were consistent. For all three years, nine of the 10 states with the highest potentially avoidable hospitalization rates and nine of the 10 states with the lowest rates were the same (see Exhibit 5).

Exhibit 5. States with lowest and highest potentially avoidable hospitalization rates, 2007 to 2009.

\begin{tabular}{ll}
\hline \multicolumn{1}{l}{ Potentially Avoidable Hospitalizations } \\
\hline 10 States with the Lowest Rates & 10 States with the Highest Rates \\
\hline California & Arkansas \\
Hawaii & Illinois \\
Idaho & Indiana \\
Maine & Kansas \\
Minnesota & Kentucky \\
New Mexico (2007-2008) / Oregon (2009) & Louisiana \\
Utah & Mississippi \\
Vermont & New Jersey \\
Washington & Ohio \\
\hline
\end{tabular}

NOTE: *States are listed in alphabetical order.

SOURCE: Chronic Condition Warehouse, 2007-2009. 


\section{Discussion}

Leading experts have suggested potentially avoidable hospitalizations lead to poor health outcomes and costly care. From 2007 to 2009, potentially avoidable hospitalizations rates remained stable, and there was virtually no change in the 10 highest- and 10 lowest-ranked states.

Over the last several years, CMS has undertaken several initiatives to reduce potentially avoidable hospitalizations. In 2012, CMS launched an initiative to reduce potentially avoidable hospitalizations among nursing facility residents, and chose seven organizations to implement a variety of programs to try and reduce unnecessary hospitalizations (Centers for Medicare \& Medicaid Services, 2013c). Previous initiatives that focused on changes to process, communication, and personnel within nursing facilities, though small in scale, reduced all hospitalizations by 17 percent to 47 percent (Kane, Keckhafer, Flood, Bershadsky, \& Siadaty, 2003; Ouslander et al., 2011). Several other CMS efforts have focused on lowering readmissions among the Medicare FFS population (Gerhardt, Yemane, Hickman, Oeschlaeger, Rollins, and Brennan, 2013). These include reporting hospital readmission rates through Hospital Compare, and funding hospitallevel improvements through the Partnership for Patients Program (Centers for Medicare \& Medicaid Services, 2013d, 2013e, 2013f). These initiatives should be monitored in the future to see if they result in reductions in these rates.

\section{Correspondence}

Misha Segal, M.B.A., Department of Health and Human Services, Centers for Medicare \& Medicaid Services, 200 Independence Avenue, Washington, D.C. 20201, misha.segal@cms.hhs.gov, Tel. 202-205-0222, Fax. 202-401-7438.

\section{Disclaimer}

The authors have been requested to report any funding sources and other affiliations that may represent a conflict of interest. The authors reported that there are no conflict of interest sources.

\section{References}

Carter, M. W. (2003). Factors associated with ambulatory care-sensitive hospitalizations among nursing home residents. Journal of Aging and Health, 15(2), 295-331. PubMed http:// dx.doi.org/10.1177/0898264303015002001

Centers for Medicare \& Medicaid Services (2013a). Data Analysis Brief: MedicareMedicaid Dual Enrollment from 2006 through 2011. Retrieved from: http://www.cms.gov/ Medicare-Medicaid-Coordination/Medicareand-Medicaid-Coordination/MedicareMedicaid-Coordination-Office/Downloads/ Dual_Enrollment_2006-2011_Final_ Document.pdf

Centers for Medicare \& Medicaid Services (2013b).

Geographic Variation Database [Online Data Resource]. Retrieved from http://www.cms. gov/Research-Statistics-Data-and-Systems/ Statistics-Trends-and-Reports/MedicareGeographic-Variation/index.html

Centers for Medicare \& Medicaid Services (2013c). Initiative to Reduce Avoidable Hospitalizations Among Nursing Facility Residents. Retrieved from http://www.innovations.cms.gov/initiatives/ rahnfr/index.html

Centers for Medicare \& Medicaid Services (2013d). Hospital Compare [Online Comparison Tool]. Retrieved from http://www.medicare.gov/ hospitalcompare 
Centers for Medicare \& Medicaid Services (2013e). Hospital Readmission Reduction Program. Retrieved from http://www.cms.gov/ Medicare/Medicare-Fee-for-Service-Payment/ AcuteInpatientPPS/Readmissions-ReductionProgram.html

Centers for Medicare and Medicaid Services). (2013f). Accountable Care Organization. Retrieved from http://www.cms.gov/Medicare/ Medicare-Fee-for-Service-Payment/ACO/ index.html

Coughlin, T. A., Waidmann, T., \& Phadera, L. (2012). Among Dual Eligibles, Identifying The Highest-Cost Individuals Could Help In Crafting More Targeted And Effective Responses. Health Affairs, 31(5), 1083-1091.PubMed http://dx.doi. org/10.1377/hlthaff.2011.0729

Gerhardt, G., Yemane, A., Hickman, P., Oeschlaeger, A., Rollins, E., \& Brennan, N. (2013). Medicare Readmission Rates Showed Meaningful Decline in 2012. Medicare \& Medicaid Research Review, 3(2), E1-E12. http://dx.doi.org/10.5600/mmrr. 003.02.b01

Grabowski, D. C., O’Malley, A., \& Barhydt, N. (2007). The Costs and Potential Savings Associated with Nursing Home Hospitalizations. Health Affairs, 26(6), 1753-1761.PubMed http:// dx.doi.org/10.1377/hlthaff.26.6.1753

Kane, R. L., Keckhafer, G., Flood, S., Bershadsky, B., \& Siadaty, M. S. (2003). The Effect of Evercare on Hospital Use. Journal of the American Geriatrics Society, 51(10), 1427-1434. PubMed http:// dx.doi.org/10.1046/j.1532-5415.2003.51461.x

Maslow, K., \& Ouslander, J. (2012). Measurement of Potentially Preventable Hospitalizations. Washington, Dc: Long-Term Quality Alliance.
Retrieved from http://www.ltqa.org/wpcontent/themes/ltqaMain/custom/images/ PreventableHospitalizations_021512_2.pdf

Ouslander, J. G., Lamb, G., Tappen, R., Herndon, L., Diaz, S., Roos, B., . . Bonner, A. (2011). Interventions to Reduce Hospitalizations from Nursing Homes: Evaluation of the INTERACT II Collaborative Quality Improvement Project. Journal of the American Geriatrics Society, 59(4), 745-753. PubMed http://dx.doi.org/10.1111/ j.1532-5415.2011.03333.x

Segal, M. (2011). Dual Eligible Beneficiaries and Potentially Avoidable Hospitalizations. Baltimore, MD: Centers for Medicare and Medicaid Services. 2013. Retrieved from: https://www. cms.gov/Research-Statistics-Data-and-Systems/ Statistics-Trends-and-Reports/Insight-Briefs/ downloads/PAHInsightBrief.pdf

Torio, C. M., Elixhauser, A., \& Andrews, R. M. (2013). Trends in Potentially Preventable Admissions Among Adults and Children, 20052010 (HCUP Statistical Brief \#151). Rockville, MD: Agency for Healthcare Research and Quality. Retrieved from http://www.hcup-us. ahrq.gov/reports/statbriefs/sb151.pdf

Walsh, E. G., Freiman, M., Haber, S., Bragg, A., Ouslander, J., \& Wiener, J. (2010). Cost Drivers for Dually Eligible Beneficiaries: Potentially Avoidable Hospitalizations from Nursing Facility, Skilled Nursing Facility, and Home and Community-Based Services Waiver Programs. Baltimore, MD: Centers for Medicare and Medicaid Services. 2013. Retrieved from: http://www.cms.gov/Research-Statistics-Dataand-Systems/Statistics-Trends-and-Reports/ Reports/downloads/costdriverstask2.pdf 